\title{
The impact of attack frequency and duration on neurocognitive processing in migraine sufferers: evidence from event-related potentials using a modified oddball paradigm
}

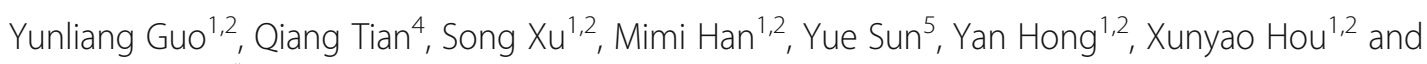
Xueping $\operatorname{Liu}^{1,2,3^{*}}$ (ID

\begin{abstract}
Background: Several studies have suggested that migraineurs suffer from neurocognitive abnormalities, but this phenomenon and exact mechanisms remain controversial. In this study, we aimed to reevaluate visual spatial attention via event-related potential (ERP) examinations and explore further correlations between ERP data and migraine characteristics.
\end{abstract}

Methods: Altogether, 25 migraine patients ( 9 males, 16 females; mean age 35.240 years) in the interictal period and 21 age-matched healthy controls ( 8 males, 13 females; mean age 35.286 years) were recruited. A modified visual oddball paradigm which contained standard, target and novel stimuli was used in the test, and amplitudes and latencies of corresponding original/difference ERP components were measured and analyzed independently.

Results: We found that P3 amplitude was markedly reduced in migraineurs. This phenomenon was further validated in analysis of difference P3 components (target minus standard and novel minus standard). Additionally, the N1 and N2 latencies elicited by novel stimulus were both delayed in patients compared with controls. Furthermore, these deviant cognitive ERPs were correlated with frequency and duration of migraine attacks.

Conclusions: These results indicated impaired visual spatial attention in migraine patients, which could be related to frequency and duration of attacks.

Keywords: Migraine, Event-related potential, P3, Impaired visual spatial attention, Attack frequency, Attack duration

\section{Background}

Migraine is one of the most common and debilitating pain disorders affecting up to $15 \%$ of the population [1], mainly causing social functioning impairment, lost productivity and a lower overall quality of life [2]. Migraine clinically manifests with recurrent attacks of headache accompanied by gastrointestinal and autonomic nervous system

\footnotetext{
*Correspondence: Liuxueping1962@163.com

'Department of Senile Neurology, Shandong Provincial Hospital Affiliated to Shandong University, Jinan 250021, Shandong, People's Republic of China ${ }^{2}$ Anti-Aging Monitoring Laboratory, Shandong Provincial Hospital Affiliated to Shandong University, Jinan 250021, Shandong, People's Republic of China Full list of author information is available at the end of the article
}

symptoms, such as nausea and photophobia [3], while the exact pathogenesis has yet to be clearly elucidated.

Some investigations suggest that migraine sufferers could have cognitive impairment, including attentional deficits, visual-spatial processing abnormalities and memory disturbances [4-6]. A nationwide retrospective cohort study has shown that migraine is associated with a higher risk of dementia [7], and an increased prevalence of stroke is found in migraineurs [8], which may lead to neurocognitive abnormalities in succession [9]. Migraine is also closely related to an elevated risk for gray matter volume reduction in frontal cortex and cingulate gyrus, deep white matter hyperintensities, and subclinical brain lesions [10-12],

(c) The Author(s). 2019 Open Access This article is distributed under the terms of the Creative Commons Attribution 4.0 International License (http://creativecommons.org/licenses/by/4.0/), which permits unrestricted use, distribution, and 
which can all result in cognitive decline [13]. However, several researches demonstrate no association existing between migraine and cognitive function [14, 15]. Hence, it is necessary to fully illustrate the relationship between migraine and cognitive processing, as well as underlying mechanisms.

Event-related potential (ERP), a kind of special evoked potential, can exhibit stimulus-related postsynaptic activity in cerebral regions and reflect corresponding neuroelectrophysiological alterations during cognitive processing. On account of objectivity, noninvasiveness and ultrahigh temporal resolution, ERP is ideally appropriate for investigating specific function of cerebral regions involved in a cognitive task, and has been applied in various neurological disorders, including migraine [16, 17]. Moreover, P3 (also called P300) is undoubtedly the most investigated wave for assessing cerebral information processing, due to its wide scalp distribution, simple recording and high reliability $[18,19]$.

As conflicting results emerge and underlying mechanisms remain unclear, it is in urgent need to further investigate neurocognitive processing in migraine sufferers. In the present work, we aimed to observe specific patterns of ERP waveforms and components elicited by a modified visual oddball paradigm in migraineurs, and explore correlations between attentional ERP components and clinical characteristics. We hypothesized that migraine patients suffered from attentive processing impairment and corresponding ERP abnormalities.

\section{Methods}

\section{Subjects and criteria}

In this study, 25 migraine patients ( 9 males, 16 females; mean age 35.240 years) were recruited from Shandong Provincial Hospital Affiliated to Shandong University, 7 with aura and 18 without aura. In addition, 21 age-matched healthy controls ( 8 males, 13 females; mean age 35.286 years) without headache also participated in the research, which were recruited from hospital staff or community.

Migraineurs were clinically diagnosed according to the beta version of the International Classification of Headache Disorders, 3rd edition (ICHD-3 beta), and were outside migraine attacks during experimental procedures. All patients received no prophylactic anti-migraine therapy, and were drug-free for at least $72 \mathrm{~h}$ prior to experiment. Patients with a history of analgesic drug overuse or addiction, mixed headache types, other neurological disorders, such as stroke and brain injuries, were excluded. We also excluded patients with abnormal findings on neurological examinations or brain morphology indicating other potential neurological diseases. Furthermore, all participants were verified to be literate, and had normal or correctedto-normal vision. Drug/substance abuse, suicide ideation and/or previous suicide attempts were exclusion criteria as well. Migraine characteristics were obtained by interview, including migraine history, frequency and duration of attacks in the previous year, and headache score which represented a scoring of the most severe migraine experienced over the past year by visual analog scale (VAS), with 0 representing no pain and 10 worst possible pain respectively.

We initially enrolled 33 migraine patients (19 females) and 23 age-matched healthy controls (14 females) in the study. Nevertheless, 8 patients (3 females) had to be excluded-two for excessive artefacts (blink and electromyographic activity) within electroencephalogram data and six for incomplete or ambiguous clinical characteristics. So only 25 migraineurs ( 16 females) were included. In terms of controls, 2 participants ( 1 female) were excluded due to technical problem during recording. Thus, we finally included 21 healthy controls ( 13 females) for further analysis.

\section{Evaluation of emotional state}

Emotional state of participants was evaluated by Self-Rating Anxiety Scale (SAS) and Self-Rating Depression Scale (SDS) as suggested in our previous study [20]. Score of SAS above 49 and score of SDS above 52 were considered as anxious and depressed, respectively [21].

\section{Stimuli and procedure}

The experiment was performed in an electrical shielded and quiet room with dim light. The subjects were seated in a comfortable armchair and were instructed to fix their eyes on a fixation cross at the center of a computer monitor (23 in.) placed $50 \mathrm{~cm}$ in front of them. A practice run was carried out to ensure that all participants understood this task, and 3 separate blocks of 501 stimuli in total were presented in random order. A modified visual oddball paradigm was used in this experiment, which consisted of standard (smaller circle, $p=0.76, n=$ 381 ), target (larger circle, $p=0.12, n=60$ ) and novel (square, $p=0.12, n=60$ ) stimuli. The duration of each stimulus was $400 \mathrm{~ms}$, and the inter-stimulus interval was fixed at $400 \mathrm{~ms}$. All subjects were not informed about the incidence of different stimuli, and were directed to discriminate larger circles (target stimuli, $n=60,20$ for each block) as accurately and quickly as possible when the paradigm was presented. Participants were required to remember the amount of larger circles they viewed in every block by mental counting and report to experimenter, so that accuracy could be calculated and recorded. Accuracy was defined as the difference between reported number and exact number, and subjects with accuracy below $90 \%$ were removed from analysis.

\section{Electroencephalogram recordings}

The electroencephalogram (EEG) of all participants was continuously recorded using $\mathrm{Ag}-\mathrm{AgCl}$ active electrodes placed in accordance with the 10-20 international 
system via Neurolab EEG/ERPs 32 Channel Amplifier. Recordings were obtained from midline $\mathrm{Fz}, \mathrm{Cz}$ and $\mathrm{Pz}$ electrode sites, each referenced to left mastoid signals (right mastoid as ordinary recording site). The electrode sites on the scalp were prepared with alcohol and scraped with scrub cream to remove cutin, thus electrode impedance could be maintained below $10 \mathrm{k} \Omega$ throughout the experiment. In addition, electrodes placed supraorbitally to the right eye and $10 \mathrm{~mm}$ from the right outer canthus were used to record eye movements and remove electrooculogram (EOG) artifacts subsequently. The sampling rate was $1000 \mathrm{~Hz}$, and the low pass filter was set at $100 \mathrm{~Hz}$.

\section{EEG data preprocessing and measurements}

We used ASA 4.9.3 software to analyze EEG data off-line. The data were re-referenced to the average of left and right mastoid signals. EOG artifacts were corrected using the method proposed by Jung et al. [22], and the bandpass filter was set at $0.1-30 \mathrm{~Hz}(24 \mathrm{~dB} /$ octave) for data preprocessing.
Then epochs were extracted from $200 \mathrm{~ms}$ pre-stimulus to $1000 \mathrm{~ms}$ post-stimulus. A - 200 to $0 \mathrm{~ms}$ pre-stimulus baseline was applied for all ERP waveform corrections and measurements. Trials with signal exceeding $\pm 100 \mu \mathrm{V}$ amplitude in any recording channel were rejected from averaging. Finally, segments of standard, target and novel stimuli were averaged separately, and single-subject waveforms were used to generate group-averaged waveforms for further analysis.

The peak amplitudes and latencies of ERP components were measured relative to the pre-stimulus baseline period. The positive peak that appeared between 300 and $500 \mathrm{~ms}$ post-stimulus was used to define the P3 component. Additionally, the negative peak between 50 and $190 \mathrm{~ms}$, the positive peak between 110 and $270 \mathrm{~ms}$, and the negative peak between 210 and $370 \mathrm{~ms}$ were used to define the N1, P2 and N2 components, respectively (see Fig. 1).

To reliably observe and better assess the target and novel effects, the difference waveforms were calculated by subtracting ERPs in response to standard stimuli from

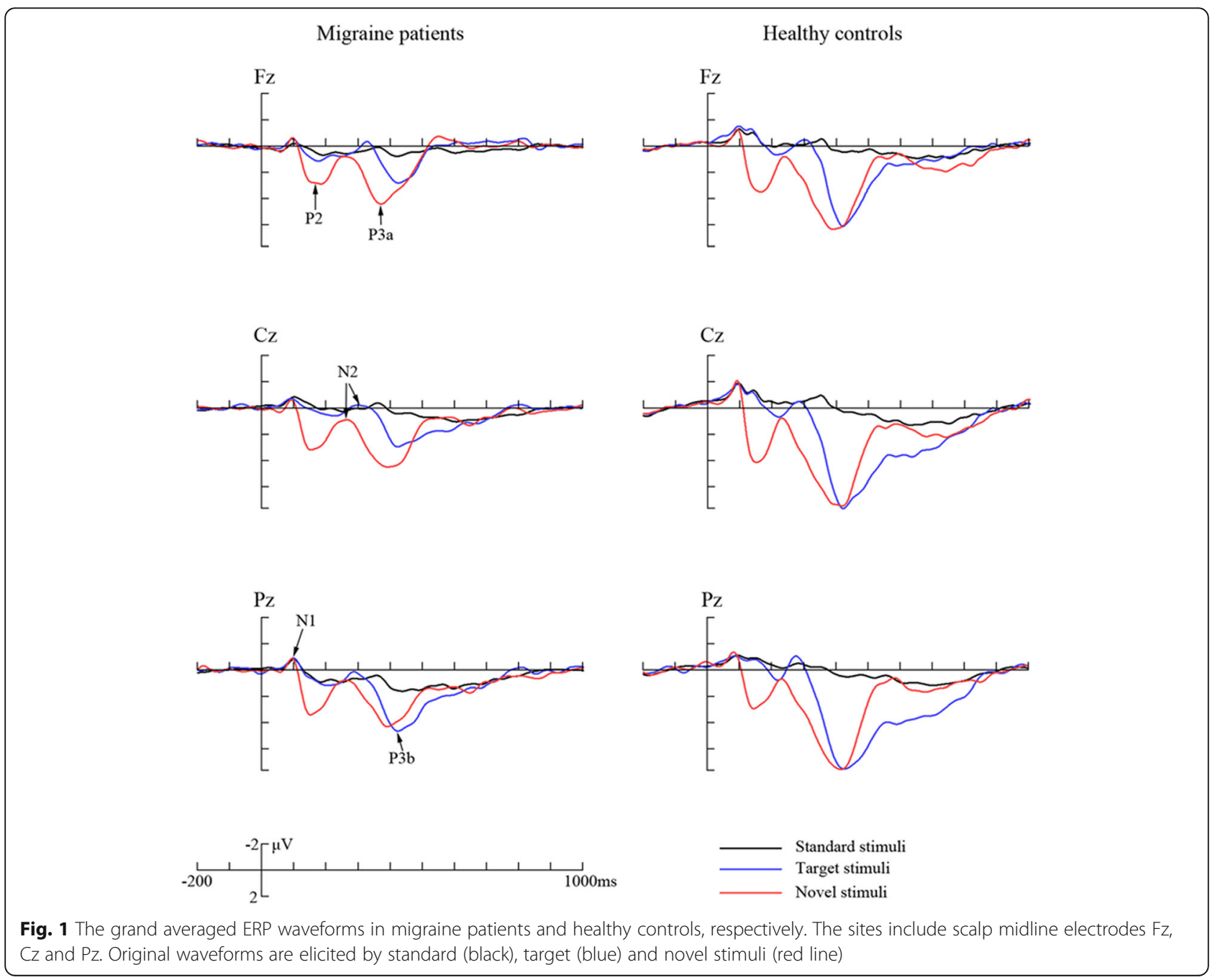


those in response to target and novel stimuli, respectively, and corresponding components were further analyzed in difference waveforms (Fig. 3).

\section{The analyses and measurements were performed blindly by the examiners \\ Statistical analysis}

Quantitative data were expressed as mean \pm standard deviation (SD). The comparisons of two groups in demographic features, emotional characteristics and behavioral performance were analyzed by Student's $t$-test for independent samples or $\chi^{2}$ test (two-tailed). Repeated-measures analysis of variance (ANOVA) was used to analyze amplitudes and latencies of original ERP components, with stimulus (standard, target and novel) and electrode $(\mathrm{Fz}, \mathrm{Cz}$ and $\mathrm{Pz})$ as within-subject factors, while with group (migraine patients vs. healthy controls) as a between-subject factor. Notably, P3 elicited by target and novel stimuli was defined as P3b and P3a components, respectively, and the method applied to analyze P3 amplitudes and latencies was same as mentioned above. For difference ERP components, repeatedmeasures ANOVA was performed with electrode $(\mathrm{Fz}, \mathrm{Cz}$ and $\mathrm{Pz}$ ) as a within-subject factor, while with group (migraine patients vs. healthy controls) as a between-subject factor. The degrees of freedom were corrected using Greenhouse-Geisser epsilon in the case of a sphericity assumption violation. Further post-hoc analysis using Bonferroni correction was conducted if necessary. Moreover, Pearson product-moment correlation coefficients $(r)$ were calculated and reported to represent correlations between clinical variables and electrophysiological data. All statistical analyses were conducted with SPSS 23.0 (SPSS Inc., Chicago, IL, USA), and results with $p<0.05$ were considered to be significant. Effect sizes of remarkable results were reported as partial eta squared $\left(\eta^{2}\right)$.

Table 1 Sample characteristics

\begin{tabular}{|c|c|c|c|c|}
\hline & Migraine patients & Healthy controls & $t / x^{2}$ & $P$ \\
\hline $\mathrm{N}$ & 25 & 21 & & \\
\hline Age, years & $35.240 \pm 11.336$ & $35.286 \pm 9.233$ & -0.014 & 0.989 \\
\hline Age range, years & $20-58$ & $24-55$ & & \\
\hline Gender, male/female & $9 / 16$ & $8 / 13$ & 0.022 & 0.883 \\
\hline Education, years & $14.320 \pm 3.484$ & $14.952 \pm 4.488$ & -0.526 & 0.602 \\
\hline Migraine frequency, times per month & $3.160 \pm 2.257$ & - & - & - \\
\hline Duration of migraine, Hours & $20.320 \pm 21.205$ & - & - & - \\
\hline History of migraine, Years & $8.010 \pm 6.522$ & - & - & - \\
\hline Headache score, 0-10 & $5.360 \pm 1.091$ & - & - & - \\
\hline SAS score & $47.520 \pm 9.575$ & $35.476 \pm 7.481$ & 4.583 & $0.000^{* * *}$ \\
\hline SDS score & $46.440 \pm 10.737$ & $35.857 \pm 8.785$ & 3.534 & $0.001^{* *}$ \\
\hline
\end{tabular}

\section{Results}

Sample characteristics

The demographic and clinical characteristics of subjects are shown in Table 1. The age, gender and education level were not significantly different between two groups (all $p>0.6$ ). In this study, migraineurs experienced 3.160 (SD 2.257) times of attacks per month, with each lasting for $20.320 \mathrm{~h}$ (SD 21.205). On average, they had been suffering from migraine for 8.010 years (SD 6.522), and average headache score was 5.360 (SD 1.091). As for emotional characteristics, assessed by SAS and SDS, migraine patients tended to be more anxious and depressed compared with healthy controls ( $p<0.001$ and $p=0.001$ respectively).

\section{Behavioral performance}

There was no remarkable difference in accuracy between two groups (migraineurs, 97.7\%; healthy controls, 98.4\%; $t=-1.244, p=0.220$ ).

\section{Characterization of original ERP data}

The grand averaged ERP waveforms are shown in Fig. 1, and analyses of multiple original ERP components are summarized in Table 2.

\section{P3 component}

Figure 2 depicts topographies of voltage distribution for P3 component elicited by standard, target (P3b) and novel stimuli (P3a). Migraineurs displayed lower P3 amplitude $(4.245 \pm 3.085 \mu \mathrm{V})$ than healthy controls $(6.360 \pm 4.746 \mu \mathrm{V}$, $F(1,44)=12.278, p=0.001$, partial $\left.\eta^{2}=0.218\right)$. Across groups, the main effect of stimulus was significant $\left(F(1.885,82.944)=50.827, p<0.001\right.$, partial $\left.\eta^{2}=0.536\right)$, with a maximum of $7.040 \mu \mathrm{V}$ for novel-elicited P3a. Furthermore, there was a remarkable group $\times$ stimulus interaction $\left(F(1.885,82.944)=6.261, p=0.003\right.$, partial $\eta^{2}=$ 0.125) (Table 2), and further post-hoc analysis revealed

Data were expressed as mean $\pm S D$

SAS Self-Rating Anxiety Scale, SDS Self-Rating Depression Scale

${ }^{* *} P<0.01,{ }^{* * *} P<0.001$ by Student's $t$-test (two-tailed) 
Table 2 Results and analyses of original ERP components

\begin{tabular}{|c|c|c|c|c|c|c|c|c|c|}
\hline \multirow{2}{*}{\multicolumn{2}{|c|}{ Original }} & \multicolumn{3}{|c|}{ Migraine patients } & \multicolumn{3}{|c|}{ Healthy controls } & \multicolumn{2}{|l|}{ Statistics } \\
\hline & & & & & & & & \multirow{2}{*}{$\begin{array}{l}\text { Group } \\
F\end{array}$} & \multirow{2}{*}{$\begin{array}{l}\text { Group } \times \text { Stimulus } \\
F\end{array}$} \\
\hline ERPs & & Standard & Target & Novel & Standard & Target & Novel & & \\
\hline \multirow[t]{4}{*}{ P3 } & Amplitude $(\mu \mathrm{V})$ & 2.243 & $4.828^{\mathrm{a}}$ & $5.665^{b}$ & 2.263 & $8.402^{a}$ & $8.416^{b}$ & $12.278^{* *}$ & $6.261^{* *}$ \\
\hline & & $(1.800)$ & $(3.001)$ & (3.180) & $(1.426)$ & (3.977) & $(4.985)$ & & \\
\hline & Latency (ms) & 426.720 & $431.920^{\mathrm{a}}$ & $405.693^{b}$ & 432.397 & $430.095^{\mathrm{a}}$ & $401.302^{b}$ & 0.001 & 0.547 \\
\hline & & (39.785) & (31.326) & (39.050) & (37.928) & (30.989) & $(37.246)$ & & \\
\hline \multirow[t]{4}{*}{$\mathrm{N} 1$} & Amplitude $(\mu \mathrm{V})$ & -1.437 & -1.623 & -2.120 & -2.093 & -2.587 & -2.941 & 3.922 & 0.206 \\
\hline & & $(1.468)$ & $(1.663)$ & $(2.367)$ & (1.319) & $(2.324)$ & $(2.353)$ & & \\
\hline & Latency (ms) & 117.653 & 115.493 & 111.027 & 122.381 & 117.714 & 99.032 & 0.080 & $4.818^{*}$ \\
\hline & & (29.340) & (32.314) & (27.180) & (30.310) & (31.819) & $(23.011)$ & & \\
\hline \multirow[t]{4}{*}{ P2 } & Amplitude $(\mu \mathrm{V})$ & 1.685 & 1.942 & 4.564 & 1.616 & 2.614 & 5.766 & 1.547 & 1.566 \\
\hline & & $(1.713)$ & $(1.831)$ & (3.550) & $(1.656)$ & (2.218) & (3.198) & & \\
\hline & Latency (ms) & 213.453 & 217.560 & 180.827 & 205.937 & 210.111 & 168.095 & 2.697 & 0.177 \\
\hline & & (34.593) & (32.282) & (39.135) & (29.831) & (29.958) & (34.486) & & \\
\hline \multirow[t]{4}{*}{ N2 } & Amplitude $(\mu \mathrm{V})$ & -0.618 & -1.086 & -0.362 & -1.470 & -1.235 & -0.207 & 0.370 & 1.484 \\
\hline & & (1.654) & (2.058) & $(2.741)$ & (2.108) & $(2.021)$ & $(2.551)$ & & \\
\hline & Latency (ms) & 303.253 & 292.440 & 269.080 & 310.635 & 281.349 & 254.016 & 1.561 & $3.407^{*}$ \\
\hline & & (32.840) & $(29.347)$ & (23.952) & (45.156) & (29.665) & $(36.216)$ & & \\
\hline
\end{tabular}

SD appeared in parenthesis of the mean

${ }^{\mathrm{a}}$ Target-elicited P3b; ${ }^{\mathrm{b}}$ novel-elicited P3a

${ }^{*} P<0.05,{ }^{*} P<0.01$ by repeated-measures ANOVA (Bonferroni correction)

marked differences in P3b and P3a amplitudes between two groups $\left(F(1,44)=16.057, p<0.001\right.$, partial $\eta^{2}=0.267$ for P3b; $F(1,44)=6.061, p=0.018$, partial $\eta^{2}=0.121$ for P3a), while not for standard stimulus $(F(1,44)=0.003, p=$ 0.958) (see Fig. 2). Additionally, we compared P3b and P3a amplitudes between groups at each electrode, and all results reached significant levels $(p s<0.04)$ except for P3a at $\mathrm{Fz}(F(1,44)=3.829, p=0.057)$. The amplitude of $\mathrm{P} 3$ showed noticeable main effect of site $(F(1.578,69.437)=$ 5.883, $p=0.008$, partial $\eta^{2}=0.118$ ). Moreover, difference between the location of recording was not observed: left (F3, C3 and P3) vs. right (F4, C4 and P4) vs. midline (Fz, $\mathrm{Cz}$ and $\mathrm{Pz})(p \mathrm{~s}>0.05)$.

In terms of P3 latency, remarkable stimulus effect $\left(F(1.852,81.479)=19.174, p<0.001\right.$, partial $\left.\eta^{2}=0.304\right)$ was obtained, of which novel-elicited P3a was fastest $(403.497 \mathrm{~ms})$. The analysis did not reveal other significant results $(p s>0.05)$.

\section{N1 component}

We did not find notable effects or interactions in N1 amplitude $(p s>0.05)$.

As shown in Table 2, although there existed no difference between groups in N1 latency $(F(1,44)=0.080, p=$ 0.779 ), the two-way interaction of group $\times$ stimulus appeared remarkable $(F(1.524,67.037)=4.818, p=0.018$, partial $\eta^{2}=0.099$ ), and post-hoc analysis discovered that the latency elicited by novel stimulus was obviously prolonged in migraineurs $(F(1,44)=4.709, p=0.035$, partial $\left.\eta^{2}=0.097\right)$, while this phenomenon was not observed for standard $(F(1,44)=0.490, p=0.488)$ and target stimuli $(F(1,44)=0.077, p=0.783)$.

\section{P2 component}

Neither group effects $(F(1,44)=1.547, p=0.220$ for amplitude; $F(1,44)=2.697, p=0.108$ for latency) nor group $\times$ stimulus interactions $(F(1.186,52.196)=1.566, p=0.219$ for amplitude; $F(1.593,70.094)=0.177, \quad p=0.788$ for latency) was observed significant in analysis of P2 component. Other effects or interactions did not reach significant levels either $(p s>0.05)$.

\section{N2 component}

No statistically significant difference was obtained in N2 amplitude ( $p \mathrm{~s}>0.05)$.

As for N2 latency, it did not differ between groups $(F(1,44)=1.561, \quad p=0.218)$, but the noticeable main effect of stimulus $(F(1.839,80.908)=9.162, p<0.001$, partial $\left.\eta^{2}=0.528\right)$ and interaction of group $\times$ stimulus $\left(F(1.839,80.908)=3.407, p=0.042\right.$, partial $\left.\eta^{2}=0.072\right)$ was found. It was noteworthy that only latency elicited by novel stimulus was markedly delayed in patients compared with controls $\left(F(1,44)=4.398, p=0.042\right.$, partial $\eta^{2}$ $=0.091$ ). 


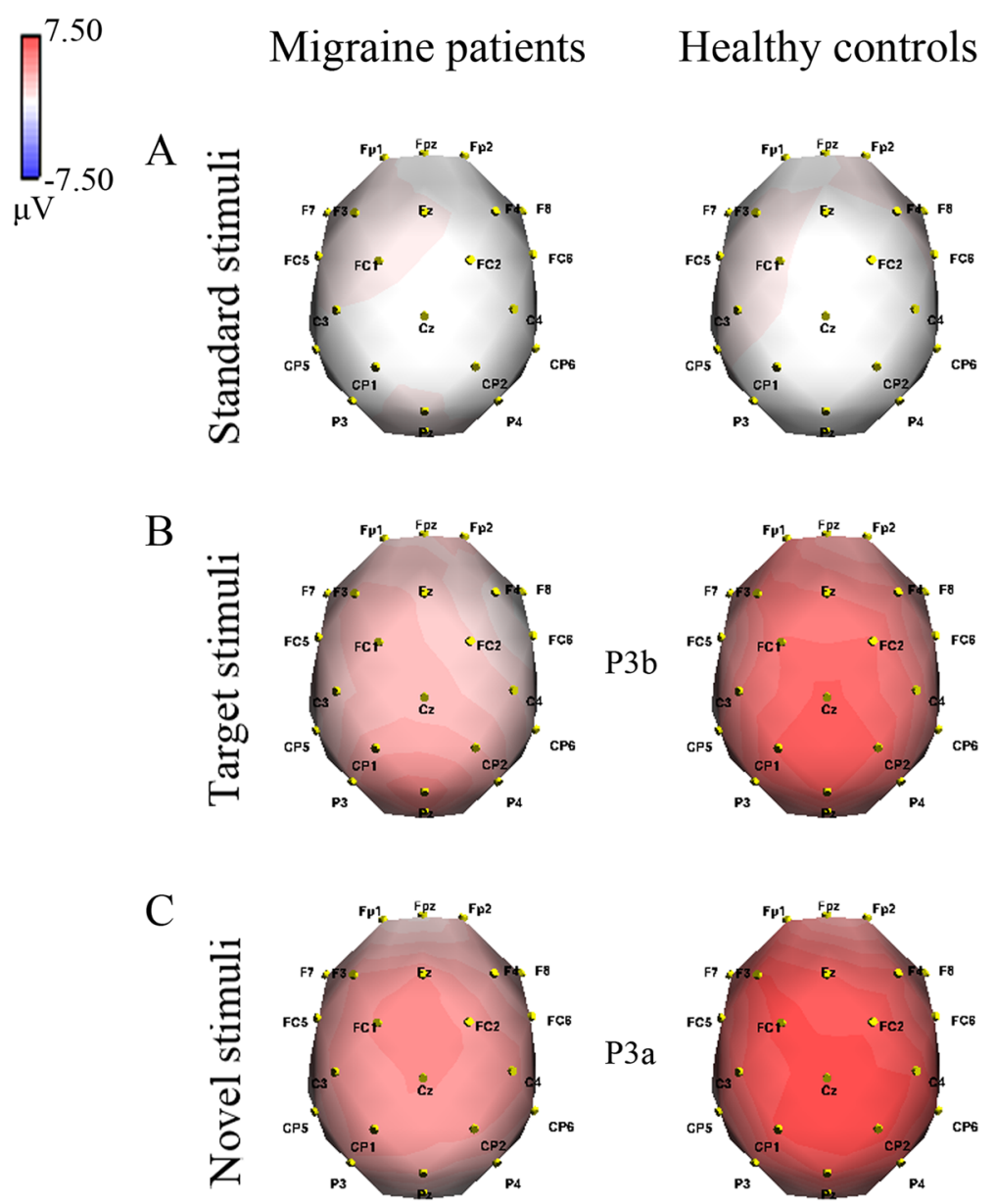

Fig. 2 Topographical voltage distributions of P3 component in migraine patients and healthy controls, respectively. P3 is elicited by different stimuli. a Standard stimuli at 425-435 ms; b target stimuli (P3b) at 430-440 ms; c novel stimuli (P3a) at 400-410 ms. Red denotes a positive and blue a negative potential

\section{Characterization of difference ERP data}

The difference waveforms are demonstrated in Fig. 3, in which P3 difference components were further analyzed (Table 3 and Fig. 4).

\section{$P 3 d_{T}$ and $P 3 d_{N}$ components}

We measured and analyzed $\mathrm{P} 3 \mathrm{~d}_{\mathrm{T}}$ (target minus standard)

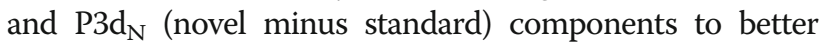
evaluate the P3 target and novel effects, respectively. The analysis of amplitudes both demonstrated significant electrode effects $\left(F(1.845,81.183)=20.346, p<0.001\right.$, partial $\eta^{2}$ $=0.316$ for $\mathrm{P}_{\mathrm{T}} ; F(1.857,81.705)=5.283, p=0.008$, partial $\eta^{2}=0.107$ for $\mathrm{P}^{2} \mathrm{~d}_{\mathrm{N}}$ ). The amplitudes were smaller for migraine patients $\left(\mathrm{P}^{2} \mathrm{~d}_{\mathrm{T}}: 4.075 \pm 1.516 \mu \mathrm{V}, \quad \mathrm{P}_{\mathrm{N}} \mathrm{d}: 5.347 \pm\right.$ $2.015 \mu \mathrm{V})$ than for healthy controls $\left(\mathrm{P} 3 \mathrm{~d}_{\mathrm{T}}: 7.955 \pm 2.787 \mu \mathrm{V}\right.$, P3d $_{\mathrm{N}}: 8.631 \pm 2.893 \mu \mathrm{V} ; F(1,44)=35.905, p<0.001$, partial

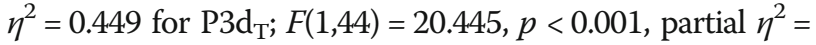
0.317 for $\left.\mathrm{P} \mathrm{d}_{\mathrm{N}}\right)$, and the significant interactions of group $\times$ electrode $\left(F(1.845,81.183)=3.416, p=0.041\right.$, partial $\eta^{2}=$ 0.072 for $\mathrm{P} 3 \mathrm{~d}_{\mathrm{T}} ; F(1.857,81.705)=3.397, p=0.042$, partial $\eta^{2}=0.072$ ) were also observed (see Table 3 and Fig. 4). Subsequent comparisons indicated that patients exhibited reduced $\mathrm{P} 3 \mathrm{~d}_{\mathrm{T}}$ and $\mathrm{P} 3 \mathrm{~d}_{\mathrm{N}}$ amplitudes in all electrodes (ps $<0.004)$.

We failed to find significant difference in latencies of P3d $_{\mathrm{T}}$ and P3d $\mathrm{d}_{\mathrm{N}}($ all $p>0.05)$.

\section{Correlations between attentional ERP components and clinical characteristics}

Based on the aforementioned results, P3 amplitude, N1 and N2 latencies elicited by different stimuli, as well as amplitudes of P3d and P3d the relationships between electrophysiological data and clinical features in migraineurs. It was discovered that P3b and $\mathrm{P}_{3} \mathrm{~d}_{\mathrm{N}}$ amplitudes showed significant, moderate and negative correlations with migraine frequency. In addition, N1 latency-novel and N2 latency-target were also positively correlated with migraine frequency. We also observed significant relationships between amplitudes of P3b, P3a and $\mathrm{P} \mathrm{d}_{\mathrm{N}}$, N1 latency-standard, N2 latency-novel and duration 


\section{A Target minus standard}
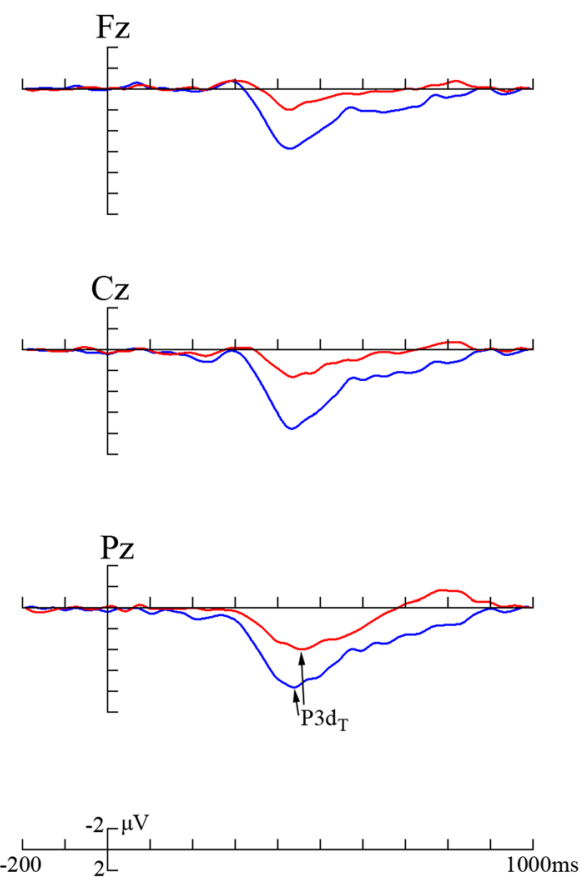

B Novel minus standard
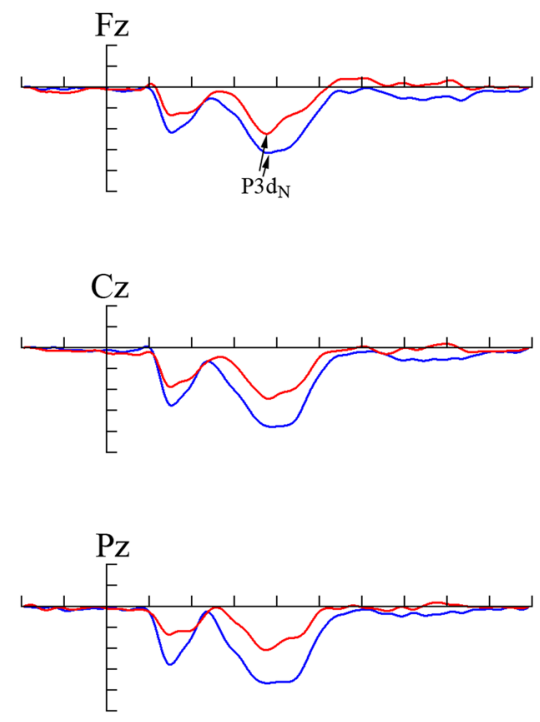

Migraine patients
Healthy controls

Fig. 3 The averaged difference waveforms in migraine patients and healthy controls, respectively. The sites include scalp midline electrodes Fz, $\mathrm{Cz}$ and Pz. a Target minus standard difference ERPs; $\mathbf{b}$ novel minus standard difference ERPs. P3d $\mathrm{d}_{\mathrm{T}}$ and P3d $\mathrm{d}_{\mathrm{N}}$ represent the P3 target and novel effects, respectively. Red line represents migraine patients and blue line healthy controls

of migraine, whereas the remaining ERP data did not correlate with migraine characteristics (Table 4).

\section{Discussion}

Migraine is the most common disabling primary headache disorder, not only for a wide range of clinical symptoms related to migraine attacks, but also for its potential cognitive disturbances [4-6, 23]. In this study, by using a three-stimulus visual oddball paradigm, reduced amplitudes of $\mathrm{P} 3, \mathrm{P} 3 \mathrm{~d}_{\mathrm{T}}$ and $\mathrm{P} 3 \mathrm{~d}_{\mathrm{N}}$ components, together with delayed $\mathrm{N} 1$ and $\mathrm{N} 2$ latencies elicited by novel stimulus were found in migraineurs. Moreover, these cognitive ERP abnormalities were directly correlated with frequency and duration of migraine attacks. The aforementioned results suggested that migraine patients might suffer from abnormalities in visual spatial attention, such as target processing, orienting responses and speed of visual information processing, especially

Table 3 Results and analyses of difference P3 components

\begin{tabular}{|c|c|c|c|c|c|c|c|c|c|}
\hline & & \multicolumn{3}{|c|}{ Migraine patients } & \multicolumn{3}{|c|}{ Healthy controls } & \multicolumn{2}{|l|}{ Statistics } \\
\hline \multicolumn{8}{|c|}{ Difference } & \multirow{2}{*}{$\begin{array}{l}\text { Group } \\
\text { F }\end{array}$} & \multirow{2}{*}{$\begin{array}{l}\text { Group } \times \text { Electrode } \\
F\end{array}$} \\
\hline ERPs & & $\mathrm{Fz}$ & $\mathrm{Cz}$ & $\mathrm{Pz}$ & $\mathrm{Fz}$ & $\mathrm{Cz}$ & $\mathrm{Pz}$ & & \\
\hline \multirow[t]{4}{*}{$\mathrm{P} 3 \mathrm{~d}_{\mathrm{T}}$} & Amplitude $(\mu \mathrm{V})$ & 3.198 & 3.702 & 5.326 & 6.689 & 8.543 & 8.633 & $35.905^{* * *}$ & $3.416^{*}$ \\
\hline & & $(1.505)$ & $(1.594)$ & $(2.373)$ & $(2.515)$ & (3.315) & $(3.473)$ & & \\
\hline & Latency (ms) & 417.560 & 430.440 & 434.720 & 422.095 & 438.333 & 423.286 & 0.002 & 1.429 \\
\hline & & (39.528) & $(34.141)$ & (38.806) & (32.695) & (31.806) & (30.798) & & \\
\hline \multirow[t]{4}{*}{$P 3 d_{N}$} & Amplitude $(\mu \mathrm{V})$ & 5.246 & 5.440 & 5.354 & 7.675 & 9.202 & 9.014 & $20.445^{* * *}$ & $3.397^{*}$ \\
\hline & & $(2.251)$ & $(2.196)$ & $(2.209)$ & $(2.904)$ & (3.294) & (3.295) & & \\
\hline & Latency (ms) & 377.960 & 384.520 & 390.320 & 387.524 & 385.429 & 397.476 & 0.549 & 0.531 \\
\hline & & (30.748) & $(37.047)$ & (34.329) & $(26.506)$ & $(27.298)$ & (31.295) & & \\
\hline
\end{tabular}

SD appeared in parenthesis of the mean

P $3 d_{T}$ and $P 3 d_{N}$ represent the P3 target and novel effects (target minus standard and novel minus standard), respectively

${ }^{*} P<0.05$, ${ }^{* *} P<0.001$ by repeated-measures ANOVA (Bonferroni correction) 


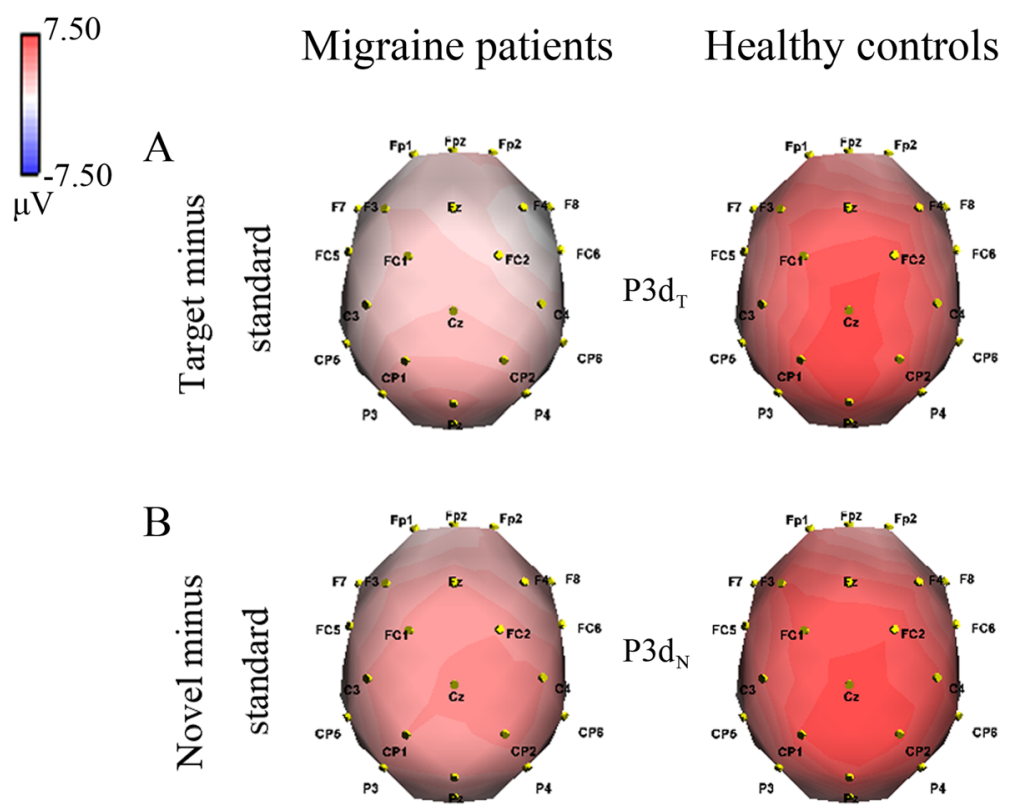

Fig. 4 Topographical voltage distributions of difference P3 components in migraine patients and healthy controls, respectively. a P3d at $420-430$ ms; b P3d at 380-395 ms. P3d and P3d $\mathrm{d}_{\mathrm{N}}$ represent the P3 target and novel effects (target minus standard and novel minus standard), respectively. Red color denotes a positive and blue color a negative potential

when triggered by infrequent stimuli, which could be potentially modulated by some clinical characteristics.

Various relatively late and positive components are defined as P3, which could be subdivided as P3b and P3a. This component is an objective index to describe characteristics of cognitive processing, with its amplitude and latency representing intensity and timing of processing respectively $[18,19]$. In our study, significant group effect and group $\times$ stimulus interaction were found in P3 amplitude, and post-hoc analysis revealed that the decreased amplitude mainly existed in target-elicited P3b and novel-elicited P3a, while the latency did not differ between groups. These findings were further validated in $\mathrm{P}_{3} \mathrm{~d}_{\mathrm{T}}$ and $\mathrm{P} 3 \mathrm{~d}_{\mathrm{N}}$ components. Consistently, other researchers observed reduced P3 amplitude in migraine patients, without any alterations in latency $[24,25]$. Nevertheless, previous study showed prolonged P3 latency rather than change of amplitude in migraineur group [26]. The discrepancies might arise from various

Table 4 Correlations between attentional ERP components and clinical characteristics in migraine patients

\begin{tabular}{lllllll}
\hline & Migraine frequency & Duration of migraine & Headache score & Migraine history & SAS score & SDS score \\
\hline Original/difference ERPs & $r(p$ value $)$ & $r(p$ value $)$ & $r(p$ value $)$ & $r(p$ value $)$ & $r(p$ value $)$ & $r(p$ value $)$ \\
P3 amplitude-standard & $-0.390(0.054)$ & $0.111(0.598)$ & $-0.165(0.432)$ & $-0.023(0.914)$ & $-0.061(0.772)$ & $-0.003(0.989)$ \\
P3 amplitude-target ${ }^{a}$ & $-0.417(0.038)^{*}$ & $-0.401(0.047)^{*}$ & $-0.212(0.309)$ & $-0.141(0.502)$ & $0.076(0.717)$ & $-0.044(0.835)$ \\
P3 amplitude-novel ${ }^{b}$ & $-0.104(0.620)$ & $-0.426(0.034)^{*}$ & $-0.176(0.400)$ & $0.031(0.882)$ & $-0.061(0.771)$ & $-0.195(0.351)$ \\
N1 latency-standard & $-0.178(0.394)$ & $0.400(0.048)^{*}$ & $0.209(0.315)$ & $0.112(0.596)$ & $-0.063(0.763)$ & $0.047(0.823)$ \\
N1 latency-target & $0.044(0.835)$ & $-0.166(0.427)$ & $0.005(0.982)$ & $0.036(0.864)$ & $0.340(0.096)$ & $0.410(0.042)^{*}$ \\
N1 latency-novel & $0.435(0.030)^{*}$ & $0.168(0.423)$ & $0.097(0.646)$ & $-0.179(0.392)$ & $0.104(0.619)$ & $0.057(0.788)$ \\
N2 latency-standard & $0.076(0.720)$ & $0.080(0.705)$ & $-0.092(0.663)$ & $0.082(0.698)$ & $0.090(0.669)$ & $-0.062(0.768)$ \\
N2 latency-target & $0.400(0.048)^{*}$ & $0.081(0.699)$ & $0.024(0.911)$ & $0.140(0.506)$ & $0.082(0.695)$ & $0.080(0.703)$ \\
N2 latency-novel & $0.001(0.995)$ & $0.398(0.049)^{*}$ & $0.329(0.108)$ & $-0.101(0.630)$ & $0.087(0.678)$ & $0.121(0.566)$ \\
P3d $d_{T}$ amplitude & $-0.056(0.791)$ & $-0.268(0.196)$ & $-0.094(0.656)$ & $-0.137(0.513)$ & $-0.018(0.933)$ & $-0.142(0.497)$ \\
P3d $d_{N}$ amplitude & $-0.405(0.044)^{*}$ & $-0.440(0.028)^{*}$ & $-0.281(0.174)$ & $0.170(0.415)$ & $-0.019(0.929)$ & $-0.085(0.685)$ \\
\hline
\end{tabular}

$r$ represents Pearson product-moment correlation coefficient

SAS Self-Rating Anxiety Scale, SDS Self-Rating Depression Scale

${ }^{\mathrm{a} P} 3 \mathrm{~b}$ amplitude; ${ }^{\mathrm{b}} \mathrm{P} 3 \mathrm{a}$ amplitude

P $3 d_{T}$ and $P 3 d_{N}$ represent the P3 target and novel effects (target minus standard and novel minus standard), respectively

${ }^{*} P<0.05$ by Pearson's correlations (two-tailed) 
methodologies used for sample selection and migraine assessment, as well as different experimental procedures. Since the amplitude of P3 mainly depends on stimulus relevance, attention allocation, memory and complexity of task [27], our findings indicated that migraineurs might have interictal impairment in visual spatial attention, such as target processing and orienting responses [28].

N1 component depicts the early sensory processing and is usually considered to reflect selective attention which represents the capability of responding to a specific stimulus [29]. N2 component, a negativity with predominant frontal-central distributions, reflects the early target selection, response preparation and conflict detection [30, 31]. Similar results were obtained from analysis of N1 and N2. We found no significant effects or interactions in $\mathrm{N} 1$ and $\mathrm{N} 2$ amplitudes. In terms of $\mathrm{N} 1$ and $\mathrm{N} 2$ latencies, the group $\times$ stimulus interactions were statistically significant, and were both markedly delayed under novel stimulus in migraine sufferers. The aforementioned results implied that migraineurs might suffer from deficits in the speed of visual information processing, especially when triggered by infrequent or unfamiliar stimulus.

P2 is predominantly distributed in frontal regions with a latency of around $200 \mathrm{~ms}$ after stimulus onset, and is believed to index automatic evaluation, task-related classification and attentional recruitment that form bases for subsequent cognitive processing $[32,33]$. The frontal area plays a vital role during these processes, and previous researches have demonstrated frontal dysfunction in migraineurs, such as reduced frontal P3a [34] and decreased gray matter density in the frontal lobe [35]. However, out of our expectations, we failed to discover any $\mathrm{P} 2$ abnormality in migraine patients. The inconsistencies could be due to different participant inclusion criteria, like broader range of ages in this experiment, and diverse evaluation protocols.

In order to illuminate relationships between attentional ERP components and clinical variables of migraine sufferers, Pearson's correlations were calculated. Almost no correlations were found between cognitive ERPs and emotional abnormalities. Meanwhile, we discovered that there were negative correlations between P3/difference P3 amplitudes and frequency and duration of attacks, and latencies of N1 and N2 were positively correlated with these characteristics. Likewise, it was verified that the poorer cognitive performance of migraineurs was related to duration and frequency of headache [26], and Calandre et al. also reported that the frequency of migraine attacks could affect visual attention [36]. The existing correlations between ERP data and migraine characteristics might arise from the fact that the experience of headache itself could modulate cognitive performance [37, 38]. Therefore, repeated migraine attacks with longer duration might result in more serious cerebral dysfunction and more severe deficits in neurocognitive processing, at least under attentional conditions.
The standardized subject selection criteria and collection of detailed migraine characteristics were included in the strengths of present study. A three-stimulus oddball paradigm with high sensitivity was used in migraineurs for the first time to investigate visual processing, and we employed difference waveforms together with voltage topographical maps to make the results more convincing. Additionally, to our knowledge, this was also the first study to systemically discuss the correlations between attentional ERP components with elaborate classification and migraine characteristics. Since it has been demonstrated that even subclinical levels of anxiety and depression may impact cognitive processing [39, 40], the influence of emotion on electrophysiological data was excluded in this study. Nevertheless, there were several limitations constraining the interpretation of our findings. Firstly, migraine characteristics, including patients' sufferings in the previous year, were obtained by interview, which might be affected by recall bias. In this study, we used a structured questionnaire to ask patients and their family members (usually spouses) who were familiar with their complaints simultaneously, and patients whose clinical characteristics were incomplete or ambiguous were excluded in order to diminish the impact of recall bias to the minimum extent. In addition, the smaller P3 amplitudes in migraine patients could also be due to a higher baseline degree of activation. However, compared with healthy controls, reduced amplitudes were only observed for target (P3b) and novel stimuli (P3a) in migraineurs, while not for standard stimuli. These findings were further validated in analysis of subtraction-derived $\mathrm{P} \mathrm{d}_{\mathrm{T}}$ (target minus standard) and P3d $\mathrm{d}_{\mathrm{N}}$ (novel minus standard) components. Therefore, it appeared that the smaller P3 amplitudes were more likely due to the impaired visual spatial attention in migraineurs rather than the higher activity for baseline. This issue needs further investigation in the future. Furthermore, the small sample size confined the investigation of the effects of age and gender, and the distinctions between migraine with and without aura were not explored either. Finally, we did not perform source analysis of deviant ERP components in migraineurs, which we would work on in the near future to uncover the underlying network-level pathology.

\section{Conclusions}

In this study, we elucidated that migraineurs had reduced P3/P3d $\mathrm{d}_{\mathrm{T}}$ (target minus standard)/P3d $\mathrm{d}_{\mathrm{N}}$ (novel minus standard) amplitudes and prolonged latencies of N1 and N2 under novel stimulus, suggesting the existence of impairment in visual spatial attention, including target processing, orienting responses and speed of visual information processing, especially in response to infrequent stimuli. These cognitive parameters were correlated with frequency and 
duration of attacks. Given this, in order to facilitate better outcomes for migraine patients, it is important to take neurocognitive processing abnormalities into account, and ERP examinations could be a sensitive method for early diagnosis.

\section{Abbreviations \\ ANOVA: Analysis of variance; EEG: Electroencephalogram; \\ EOG: Electrooculogram; ERP: Event-related potential; ICHD-3 beta: Beta version of the International Classification of Headache Disorders, 3rd edition; P3 $\mathrm{d}_{\mathrm{N}}$ : P3 novel effect (novel minus standard); P3d $\mathrm{d}_{T}$ : P3 target effect (target minus standard); $r$ : Pearson product-moment correlation coefficients; SAS: Self-Rating Anxiety Scale; SD: Standard Deviation; SDS: Self-Rating Depression Scale; VAS: Visual analog scale; $\eta^{2}$ : Partial eta squared}

\section{Acknowledgements}

We would like to thank all subjects who participated in this study. We also thanked Jing Zhang, Department of Neurology (Shandong Provincial Hospital Affiliated to Shandong University, Shandong, China) for helping to edit the manuscript to conform to submission requirements.

\section{Funding}

This research did not receive any specific grant from funding agencies in the public, commercial, or not-for-profit sectors.

\section{Availability of data and materials}

The data supporting the conclusions of this article are held electronically in Department of.

Senile Neurology, Shandong Provincial Hospital Affiliated to Shandong University, Shandong,

China. They are still being analyzed and will be made publicly available finally. The datasets used and/or analyzed during the current study are available from the corresponding author on reasonable request.

\section{Authors' contributions}

YG, QT, SX, MH, YS, YH and XH carried out the studies. YG took part in the study design, preprocessed and measured EEG data, performed the statistical analysis and drafted the manuscript. QT, SX, MH and YS helped to record subjects, collect the needed information and revise the manuscript. Professor XL participated in the experimental design, participant recruitment, discussion of results and manuscript revision. All authors read and approved this final manuscript submitted.

\section{Ethics approval and consent to participate}

The study protocol was approved by the Ethical Committee of Shandong Provincial Hospital Affiliated to Shandong University (Approval No. 2018-215), which was in accordance with the ethical principles of the Declaration of Helsinki. We obtained written and informed consent from all participants before commencement of the test.

\section{Consent for publication}

Not applicable.

\section{Competing interests}

The authors declare that they have no competing interests.

\section{Publisher's Note}

Springer Nature remains neutral with regard to jurisdictional claims in published maps and institutional affiliations.

\section{Author details}

'Department of Senile Neurology, Shandong Provincial Hospital Affiliated to Shandong University, Jinan 250021, Shandong, People's Republic of China ${ }^{2}$ Anti-Aging Monitoring Laboratory, Shandong Provincial Hospital Affiliated to Shandong University, Jinan 250021, Shandong, People's Republic of China. ${ }^{3}$ Department of Anti-Aging, Shandong Provincial Hospital Affiliated to Shandong University, Jinan 250021, Shandong, People's Republic of China. ${ }^{4}$ Department of Geriatrics, Taian City Central Hospital, Taian 271000 Shandong, People's Republic of China. ${ }^{5}$ Department of Neurology, Affiliated
Hospital of Shandong Academy of Medical Science, Jinan 250031, Shandong, People's Republic of China.

Received: 25 January 2019 Accepted: 15 April 2019

Published online: 27 April 2019

\section{References}

1. Stovner L, Hagen $K$, Jensen R, Katsarava Z, Lipton R, Scher A, et al. The global burden of headache: a documentation of headache prevalence and disability worldwide. Cephalalgia. 2007;27:193-210.

2. Terwindt GM, Ferrari MD, Tijhuis M, Groenen SM, Picavet HS, Launer LJ. The impact of migraine on quality of life in the general population: the GEM study. Neurology. 2000:55:624-9.

3. Buse DC, Rupnow MF, Lipton RB. Assessing and managing all aspects of migraine: migraine attacks, migraine-related functional impairment, common comorbidities, and quality of life. Mayo Clin Proc. 2009;84:422-35.

4. Mickleborough MJ, Hayward J, Chapman C, Chung J, Handy TC. Reflexive attentional orienting in migraineurs: the behavioral implications of hyperexcitable visual cortex. Cephalalgia. 2011;31:1642-51.

5. Coleston DM, Chronicle E, Ruddock KH, Kennard C. Precortical dysfunction of spatial and temporal visual processing in migraine. J Neurol Neurosurg Psychiatry. 1994:57:1208-11.

6. Le Pira F, Zappala G, Giuffrida S, Lo Bartolo ML, Reggio E, Morana R, et al. Memory disturbances in migraine with and without aura: a strategy problem? Cephalalgia. 2000;20:475-8.

7. Chuang CS, Lin CL, Lin MC, Sung FC, Kao CH. Migraine and risk of dementia: a nationwide retrospective cohort study. Neuroepidemiology. 2013;41:139-45.

8. Guidetti D, Rota E, Morelli N, Immovilli P. Migraine and stroke: "vascular" comorbidity. Front Neurol. 2014;5:193.

9. Leys D, Henon H, Mackowiak-Cordoliani MA, Pasquier F. Poststroke dementia. Lancet Neurol. 2005:4:752-9.

10. Jia Z, Yu S. Grey matter alterations in migraine: a systematic review and meta-analysis. Neuroimage Clin. 2017;14:130-40.

11. Cheng CY, Cheng HM, Chen SP, Chung CP, Lin YY, Hu HH, et al. White matter hyperintensities in migraine: clinical significance and central pulsatile hemodynamic correlates. Cephalalgia. 2017. https://doi.org/10.1177/ 0333102417728751.

12. Kruit MC, van Buchem MA, Hofman PA, Bakkers JT, Terwindt GM, Ferrari $M D$, et al. Migraine as a risk factor for subclinical brain lesions. JAMA. 2004;291:427-34.

13. Rist PM, Kurth T. Migraine and cognitive decline: a topical review. Headache. 2013;53:589-98

14. Pearson AJ, Chronicle EP, Maylor EA, Bruce LA. Cognitive function is not impaired in people with a long history of migraine: a blinded study. Cephalalgia. 2006;26:74-80

15. Jelicic M, van Boxtel MP, Houx PJ, Jolles J. Does migraine headache affect cognitive function in the elderly? Report from the Maastricht aging study (MAAS). Headache. 2000:40:715-9.

16. Morlet D, Demarquay G, Brudon F, Fischer C, Caclin A. Attention orienting dysfunction with preserved automatic auditory change detection in migraine. Clin Neurophysiol. 2014;125:500-11.

17. de Tommaso M, Guido M, Libro G, Losito L, Difruscolo O, Sardaro M, et al. Interictal lack of habituation of mismatch negativity in migraine. Cephalalgia. 2004;24:663-8

18. Polich J. Updating P300: an integrative theory of P3a and P3b. Clin Neurophysiol. 2007;118:2128-48.

19. Picton TW. The P300 wave of the human event-related potential. J Clin Neurophysiol. 1992;9:456-79.

20. Han M, Hou X, Xu S, Hong $Y$, Chen J, Ma Y, et al. Selective attention network impairment during the interictal period of migraine without aura. J Clin Neurosci. 2019:60:73-8.

21. Chen J, Chen C, Zhi S. Retrospective comparison of cognitive behavioral therapy and symptom-specific medication to treat anxiety and depression in throat cancer patients after laryngectomy. Shanghai Arch Psychiatry. 2014:26:95-100.

22. Jung TP, Makeig S, Humphries C, Lee TW, McKeown MJ, Iragui V, et al. Removing electroencephalographic artifacts by blind source separation. Psychophysiology. 2000:37:163-78.

23. de Araujo CM, Barbosa IG, Lemos SMA, Domingues RB, Teixeira AL. Cognitive impairment in migraine: a systematic review. Dement Neuropsychol. 2012;6:74-9. 
24. Wang R, Dong Z, Chen X, Zhang M, Yang F, Zhang X, et al. Gender differences of cognitive function in migraine patients: evidence from eventrelated potentials using the oddball paradigm. J Headache Pain. 2014;15:6.

25. Chen W, Shen $X$, Liu X, Luo B, Liu Y, Yu R, et al. Passive paradigm singletone elicited ERPs in tension-type headaches and migraine. Cephalalgia. 2007;27:139-44.

26. Huang L, Juan Dong $H$, Wang $X$, Wang $Y$, Xiao Z. Duration and frequency of migraines affect cognitive function: evidence from neuropsychological tests and event-related potentials. J Headache Pain. 2017;18:54.

27. Kok A. On the utility of $\mathrm{P} 3$ amplitude as a measure of processing capacity. Psychophysiology. 2001;38:557-77.

28. Polich J, Kok A. Cognitive and biological determinants of P300: an integrative review. Biol Psychol. 1995;41:103-46.

29. Mangun GR. Neural mechanisms of visual selective attention. Psychophysiology. 1995;32:4-18.

30. Jamadar S, Hughes M, Fulham WR, Michie PT, Karayanidis F. The spatial and temporal dynamics of anticipatory preparation and response inhibition in task-switching. Neuroimage. 2010;51:432-49.

31. Nieuwenhuis S, Yeung N, van den Wildenberg W, Ridderinkhof KR. Electrophysiological correlates of anterior cingulate function in a go/no-go task: effects of response conflict and trial type frequency. Cogn Affect Behav Neurosci. 2003;3:17-26.

32. Potts GF. An ERP index of task relevance evaluation of visual stimuli. Brain Cogn. 2004;56:5-13.

33. Carretie L, Mercado F, Tapia M, Hinojosa JA. Emotion, attention, and the 'negativity bias', studied through event-related potentials. Int J Psychophysiol. 2001;41:75-85.

34. Koo YS, Ko D, Lee GT, Oh K, Kim MS, Kim KH, et al. Reduced frontal P3a amplitude in migraine patients during the pain-free period. J Clin Neurol. 2013:9:43-50.

35. Rocca MA, Ceccarelli A, Falini A, Colombo B, Tortorella P, Bernasconi L, et al. Brain gray matter changes in migraine patients with T2-visible lesions: a 3-T MRI study. Stroke. 2006;37:1765-70.

36. Calandre EP, Bembibre J, Arnedo ML, Becerra D. Cognitive disturbances and regional cerebral blood flow abnormalities in migraine patients: their relationship with the clinical manifestations of the illness. Cephalalgia. 2002;22:291-302.

37. Houlihan ME, McGrath PJ, Connolly JF, Stroink G, Allen Finley G, Dick B, et al. Assessing the effect of pain on demands for attentional resources using ERPs. Int J Psychophysiol. 2004;51:181-7.

38. Parisi P, Verrotti A, Paolino MC, Urbano A, Bernabucci M, Castaldo R, et al. Headache and cognitive profile in children: a cross-sectional controlled study. J Headache Pain. 2010;11:45-51.

39. Wetherell JL, Reynolds CA, Gatz M, Pedersen NL. Anxiety, cognitive performance, and cognitive decline in normal aging. J Gerontol B Psychol Sci Soc Sci. 2002;57:P246-55.

40. Delle-Vigne D, Kornreich C, Verbanck P, Campanella S. The P300 component wave reveals differences in subclinical anxious-depressive states during bimodal oddball tasks: an effect of stimulus congruence. Clin Neurophysiol. 2015;126:2108-23.

\section{Ready to submit your research? Choose BMC and benefit from:}

- fast, convenient online submission

- thorough peer review by experienced researchers in your field

- rapid publication on acceptance

- support for research data, including large and complex data types

- gold Open Access which fosters wider collaboration and increased citations

- maximum visibility for your research: over $100 \mathrm{M}$ website views per year

At $\mathrm{BMC}$, research is always in progress.

Learn more biomedcentral.com/submissions 